\title{
Arteria Subclavia Derecha Retroesofágica: Reporte de Caso
}

\author{
Right Retroesophageal Subclavian Artery: Case Report
}

Ana Barriga \& Gerardo Méndez

\begin{abstract}
BARRIGA, A. \& MÉNDEZ, G. Arteria subclavia derecha retroesofágica: reporte de caso. Int. J. Morphol., 37(3):821-824, 2019.
RESUMEN: Durante el desarrollo embrionario suelen ocurrir anomalías a nivel del arco aórtico primordial. Una de estas es la arteria subclavia retroesofágica derecha (ASDR) la cual es un defecto frecuente de los arcos aórticos embriológicos y se debe a la interrupción del cuarto arco aórtico (AO) derecho entre las depresiones para la arteria carótida común y la arteria subclavia. Las variaciones anatómicas y morfológicas del $\mathrm{AO}$ y sus ramas son significativas para los procedimientos diagnósticos y quirúrgicos en el tórax y el cuello. Reportar una ASDR encontrada durante disección, correlacionando esta con sus implicancias clínicas. De un cadáver de sexo femenino, de edad y causa de muerte desconocida, fijado con formaldehído al $10 \%$ y aguada destilada. Se realizó disección del corazón y los grandes vasos, en especial el AO, desarrollado según la técnica convencional, para remover el órgano luego de disecar el pericardio y disecar la arteria aorta ascendente y el AO, preservando plenamente su configuración externa y sus ramas colaterales, donde se encontró laASDR. La ASDR presentó un diámetro en su origen de 12,13 mm. La longitud existente entre la ASDR y la Arteria carótida común Derecha (ACCD) fue de 43,84 mm. El diámetro del esófago a nivel cefálico y caudal de la ASDR alcanzó valores de 17,59 mm y 13,82 mm respectivamente. Por su parte los diámetros de la tráquea a nivel cefálico y caudal a la ASDR, fueron respectivamente 22,12 mm y $13,30 \mathrm{~mm}$. El conocimiento de esta variante anatómica resulta de gran interés a la hora de interpretar y orientar el diagnóstico de las posibles causas de una disfagia asociada a una arteria subclavia retroesofágica y fundamenta aún más la importancia del estudio mediante disección.
\end{abstract}

PALABRAS CLAVE: Arteria subclavia; Arteria subclavia aberrante; Variación anatómica.

\section{INTRODUCCIÓN}

El arco aórtico (AO) generalmente desprende tres ramas: el tronco braquiocefálico (desde el que se origina la arteria subclavia derecha (ASD) y la arteria carótida común derecha (ACCD), la arteria carótida común Izquierda (ACCI) y, por último, la arteria subclavia izquierda (ASI), desde la derecha a la izquierda (Leite et al., 2017). El AO se forma durante la vida intrauterina del saco aórtico como vaso embrionario, así mismo, varios vasos aórticos como las aortas dorsales -pares se fusionan para formar un solo vaso que en el adulto se conoce como aorta (Duque Parra et al., 2012).

Varias anomalías vasculares ocurren generalmente como resultado del fracaso en el desarrollo congénito del arco aórtico primordial (Duque et al.). Una arteria subclavia retroesofágica derecha (ASDR) es un defecto frecuente de los arcos aórticos embriológicos. Es la variación del arco aórtico con una incidencia reportada entre el 0,4 y el 1,8\% de la población (Leite et al.; Ocaya, 2015). Esta variación se debe a la interrupción del cuarto AO derecho entre las depresiones para la arteria carótida común y la arteria subclavia, mientras que el cuarto arco izquierdo permanece intacto (Duque et al.). Se produce una regresión de la porción proximal de la arteria subclavia derecha y el AO y la arteria subclavia retroesofágica persiste (Ocaya). Adachi describió por primera vez este patrón de ramificación clasificándolo como tipo $\mathrm{G}$, en el cual la arteria subclavia derecha se encontró con orientación retroesofágica en el $0.2 \%$ de 516 cadáveres japoneses (Ocaya).

Las variaciones del AO y sus ramas son bien conocidas en la literatura (Inzunza \& Burdiles, 2010; Leite et al.). El estudio de las variaciones anatómicas es relevante para quienes practicamos las ciencias morfológicas y para quienes trabajan en el diagnóstico de imágenes y en cirugía. Si bien muchas de estas variaciones pueden pasar desapercibidas durante la vida de un sujeto, en la mayoría de ellas existen implicancias de interés clínico que vale la pena estudiar (Inzunza \& Burdiles).

Las variaciones anatómicas y morfológicas del arco aórtico y sus ramas son significativas para los procedimientos diagnósticos y quirúrgicos en el tórax y el cuello (Ocaya). 
La observación accidental de la ASDR en un cadáver de sexo femenino utilizado para fines docentes en el Departamento de Anatomía del instituto de Anatomía, Histología y Patología de la Universidad Austral de Chile. Resulta especialmente atractivo por las implicaciones morfológicas y clínicas que permiten su explicación como variación anatómica.

\section{MATERIAL Y MÉTODO}

De un cadáver de sexo femenino, de edad y causa de muerte desconocida, se extrajo el bloque visceral comprendido desde la faringe hasta el esfínter anal incluyendo vísceras del aparato genitourinario, donado en 2015 por la unidad de Anatomía Patológica del Hospital Base de Valdivia al Instituto de Anatomía, Histología y Patología de la Facultad de Medicina de la Universidad Austral de Chile, para ser utilizado con fines docentes. Posteriormente este bloque visceral fue fijado con formaldehído al $10 \%$ y aguada destilada. Luego de seis meses en esta solución (lo mínimo para fijar de mejor manera los tejidos) se lavó y se mantuvo en agua corriente para comenzar la disección propiamente del corazón y los grandes vasos, en especial el AO. La disección se desarrolló según la técnica convencional, para remover el órgano luego de disecar el pericardio y disecar la arteria aorta ascendente y el AO, preservando plenamente su configuración externa y sus ramas colaterales, que posteriormente se identificaron y se procedió a rotular (Figs. 1 y 2). Se encontró a la arteria subclavia Derecha con orientación retroesofágica.

\section{RESULTADOS}

La arteria carótida común derecha (ACCD), nacida directamente desde el arco aórtico, presentó un diámetro inicial de 9,64 mm. Por su parte la arteria carótida común izquierda (ACCI) mostró un diámetro de 9,40 mm. Respecto de la ASDR presentó un diámetro en su origen de $12,13 \mathrm{~mm}$. En tanto la arteria subclavia izquierda obtuvo un diámetro de $10,9 \mathrm{~mm}$ a nivel del AO. La longitud existente entre la ASDR y la ACCD fue de 43,84 mm. El diámetro del
$\mathrm{AO}$ al inicio de la primera rama (ACCD) que emerge de él, fue de 20,24 mm y al término de la última emergencia que nace del arco (ASDR) fue de $16,91 \mathrm{~mm}$. El diámetro del esófago a nivel cefálico y caudal de la ASDR alcanzó valores de 17,59 mm y 13,82 mm respectivamente. Por su parte los diámetros de la tráquea a nivel cefálico y caudal a la ASDR, fueron respectivamente $22,12 \mathrm{~mm}$ y $13,30 \mathrm{~mm}$.

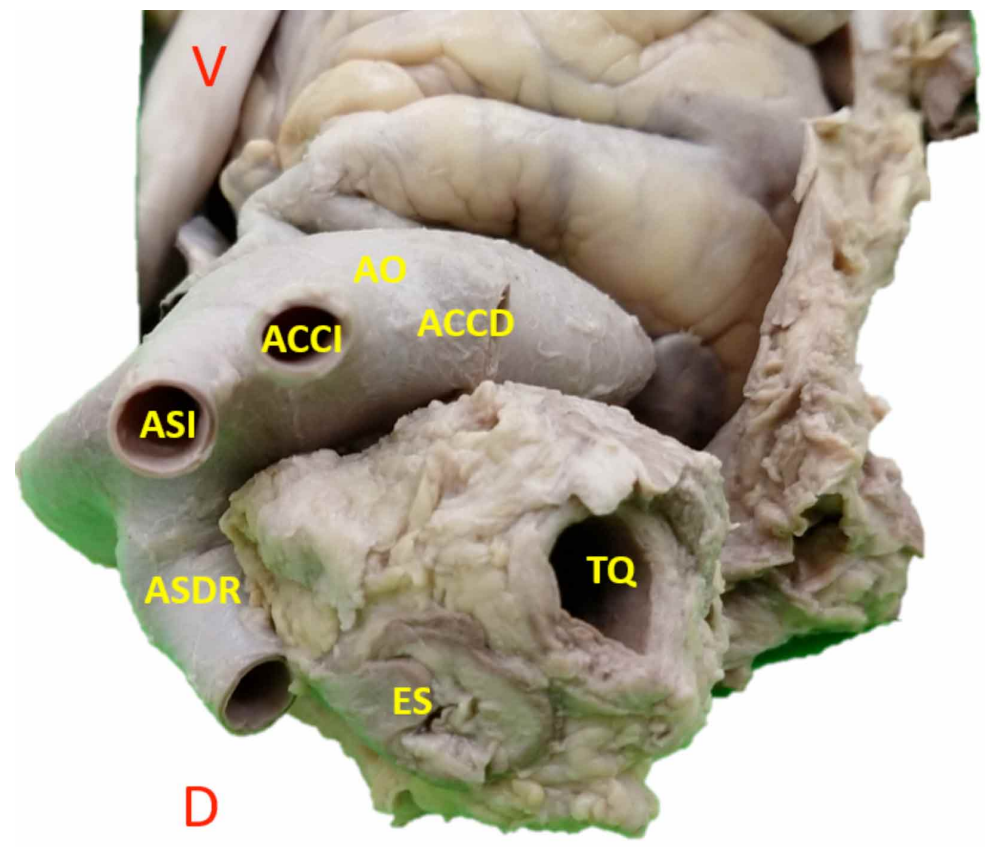

Fig. 1. Vista superior; V, ventral; D, dorsal; AO, arco aórtico; ACCD, arteria carótida común derecha; ACCI, arteria carótida común izquierda; ASI, Arteria subclavia izquierda; ASDR, arteria subclavia derecha retroesofágica; ES, esofágo; TQ, Tráquea.

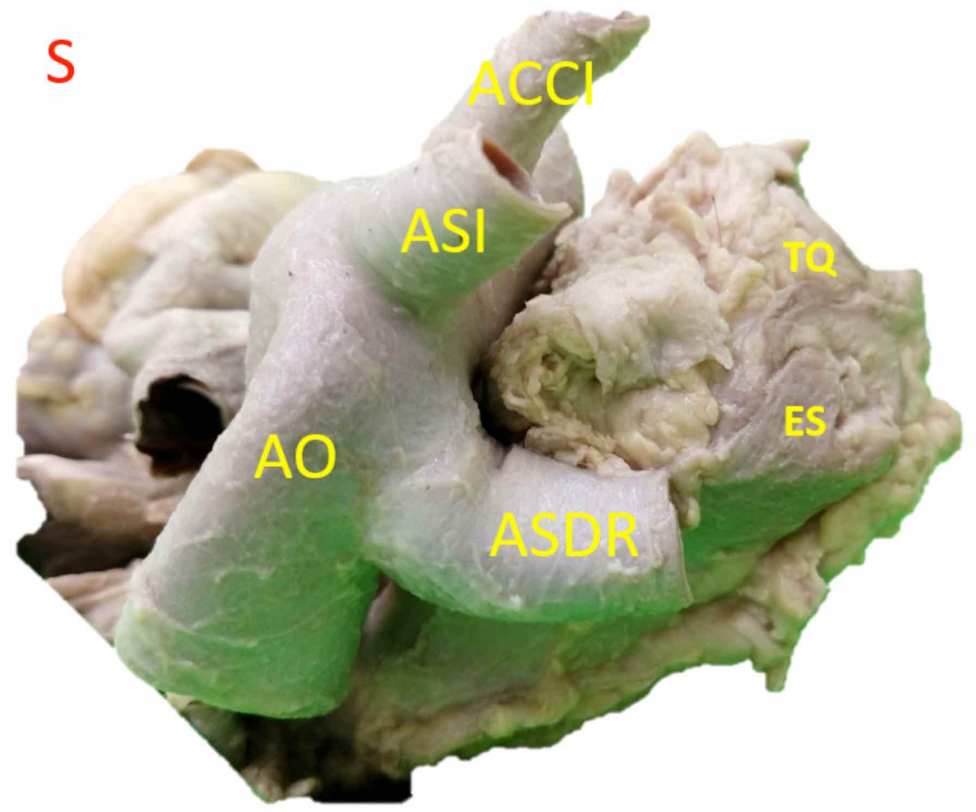

Fig. 2. Vista lateral derecha; S, superior; ACCI, arteria carótida común izquierda; ASI, Arteria subclavia izquierda; ASDR, arteria subclavia derecha retroesofágica; AO, Arco aórtico; ES, esofágo.TQ, Tráquea. 


\section{DISCUSIÓN}

Los múltiples cambios que sufre el patrón de simetría vascular de los arcos aórticos para desarrollar el modelo arterial asimétrico del adulto, implican un complejo proceso de regresiones y remodelaciones. Alteraciones en estas transformaciones pueden provocar variaciones anatómicas complejas, las que si bien pueden pasar desapercibidas durante el período fetal también pueden llegar a ser incompatibles con la vida. En este contexto, las variaciones del AO ofrecen una gran variabilidad de presentación, siendo la mayoría de ellas detectadas incidentalmente (Inzunza \& Burdiles). los resultados en cuanto al calibre de esta variación son similares a los encontrados por Inzunza \& Burdiles, además la disminución en el diámetro del esófago y la tráquea en caudal luego de la compresión por parte de la ASDR.

De entre todas estas anomalías congénitas el arco aórtico con ASDR también conocida como «arteria lusoria», es la más frecuente, encontrándose presente en un $0,5-2 \%$ de la población. Esta anomalía es el resultado de la obliteración del cuarto AO entre la ACCD y la ASDR (Duque et al.; Leite et al.; Simón-Yarza et al., 2011). En esta variante anatómica nacen del cayado aórtico, consecutivamente, las ACCD, arteria carótida común izquierda (ACCI), arteria subclavia izquierda (ASI) y subclavia derecha. ésta última atraviesa la línea media con un trayecto retroesofágico en el $85 \%$ de los casos, lo que en ocasiones produce la llamada «disfagia lusoria» al quedar comprimido el esófago entre la tráquea y la arteria subclavia aberrante (Simón-Yarza et al.). Algieri et al. (2008) concluyen además que esta variación es altamente prevalente en individuos con síndrome de Down, en los que se reportan arterias subclavias retroesofágicas hasta en el $37 \%$, sumándose a lo que concluye Inzunza \& Burdiles, donde reportan que la arteria subclavia derecha aberrante de trayecto retroesofágico es frecuentemente observada en mujeres y en niños con síndrome de Down. Además, algunos estudios han asociado a esta anomalía con el desarrollo de patologías trombo-isquémica de la extremidad superior derecha (Akers et al., 1991; Boas et al., 2002; Inzunza \& Burdiles).

La gran mayoría de los pacientes con arteria subclavia retroesofágica se encuentran asintomáticos, siendo el diagnóstico un hallazgo casual al realizar una prueba de imagen por otros motivos. En caso de disfagia, su manejo clínico depende de la severidad de los síntomas. En situaciones de disfagia leve o moderada un tratamiento conservador modificando la dieta y administrando fármacos procinéticos puede ser suficiente (Reece et al.,
2010). El tratamiento quirúrgico está reservado para aquellos casos en que la disfagia sea severa o el riesgo de rotura del aneurisma sea elevado y consiste, habitualmente, en la resección de la arteria subclavia aberrante mediante esternotomía media o toracotomía y su anastomosis a la arteria carótida común ipsilateral (Myers et al., 2010; Simón-Yarza et al.).

El conocimiento de esta variante anatómica resulta de gran interés a la hora de interpretar y orientar el diagnóstico de las posibles causas de un ensanchamiento mediastínico superior, además de ser detectado de manera incidental en exámenes imagenológicos de tórax tanto en personas asintomáticas como en pacientes que acuden al médico por disfagia. La comprensión de este tipo de variaciones abordadas desde el punto de vista morfológico ante eventuales diagnósticos, demuestra lo relevante que es el estudio de la anatomía humana mediante disección.

BARRIGA, A. \& MÉNDEZ, G. Right retroesophageal subclavian artery: case report. Int. J. Morphol., 37(3):821-824, 2019.

SUMMARY: During embryonic development, anomalies usually occur at the primordial aortic arch (AA) level. One of these is the right retroesophageal subclavian artery (RSA). The anatomical and morphological variations of AA and its branches are significant for diagnostic and surgical procedures in the thorax and neck. The objective of the study was to report an RSA found during the dissection, correlating this with the corresponding clinical implications. A human female cadaver of unknown age and cause of death was used and conserved in $10 \%$ formaldehyde and distilled wash. The dissection of the heart and large vessels was performed. There was particular emphasis one the $\mathrm{AA}$, and development according to conventional techniques, removing the organ after dissecting the pericardium and dissecting the ascending aorta and the AA. External function and its collateral branches were fully preserved, where the RSA was located. The RSA presented a diameter at its origin of $12.13 \mathrm{~mm}$. The length between the RSA and the common right carotid artery (CRCA) was 43.84 $\mathrm{mm}$. The diameter of the esophagus at the cephalic and caudal level of the RSA has values of $17.59 \mathrm{~mm}$ and $13.82 \mathrm{~mm}$ respectively. Furthermore, the diameters of the trachea at cephalic and caudal level to the RSA, respectively, were 22.12 $\mathrm{mm}$ and $13.30 \mathrm{~mm}$. Knowledge of this anatomical variant is of great interest when interpreting and guiding the diagnosis of potential causes of a dysphagia associated with a retroesophageal subclavian artery, and is even more important during dissection studies.

KEY WORDS: Subclavian Artery; Aberrant subclavian artery; Anatomic Variation. 


\section{REFERENCIAS BIBLIOGRÁFICAS}

Akers, D. L. Jr.; Fowl, R. J.; Plettner, J. \& Kempczinski, R. F. Complications of anomalous origin of the right subclavian artery: case report and review of the literature. Ann. Vasc. Surg ., 5(4):385-8, 1991.

Algieri, R. D.; Mazzoglio y Nabar, M. J. \& Ferrante, M. S. Retroesophageal variation of the aortic arch. Int. J. Morphol., 26(2):337-43, 2008.

Boas, N.; Desmoucelle, F.; Bernadet, V. \& Franceschi, J. C. Rare cause of acute ischemia of the right upper extremity: thrombosis of a retroesophageal subclavian artery. Ann. Vasc. Surg., 16(3):387-90, 2002.

Duque Parra, J. E.; Estrada Pantoja, S. L. \& Montenegro, A. A. Aortic arch variant in human cadaver. Int. J. Morphol., 30(4):1458-60, 2012.

Inzunza, O. \& Burdiles, A. Aberrant subclavian artery. Int. J. Morphol., 28(4):1215-9, 2010.

Leite, T. F. O.; Pires, L. A. S.; Cisne, R.; Babinski, M.A. \& Chagas, C.A.A. Clinical discussion of the arteria lusoria: a case report. J. Vasc. Bras., 16(4):339-42, 2017.

Myers, P. O.; Fasel, J. H.; Kalangos, A. \& Gailloud, P. Arteria lusoria: developmental anatomy, clinical, radiological and surgical aspects. Ann. Cardiol. Angeiol. (Paris), 59(3):147-54, 2010.

Ocaya, A. Retroesophageal right subclavian artery: a case report and review of the literature. Afr. Health Sci., 15(3):1034-7, 2015.

Reece, T. B.; Wallen, T. J.; Jagasia, D. H.; Bavaria, J. E. \& Szeto, W. Y. Open thoracic aortic repair for dysphagia in patients with Kommerell's diverticulum and right-sided aortic arch with aberrant left subclavian artery. J. Card. Surg., 25(1):62-4, 2010.

Simón-Yarza, I.; Viteri-Ramírez, G.; Etxano, J.; Slon, P.; Ferreira, M. \& Bastarrika, G. Arco aórtico derecho, divertículo de Kommerell y arteria subclavia izquierda aberrante. An. Sist. Sanit. Navar., 34(2):295-300, 2011.
Dirección para correspondencia:

Ana Barriga $\mathrm{K}$.

Instituto Anatomía, Histología y Patología

Facultad de Medicina

Universidad Austral de Chile

Valdivia

CHILE

Email: anabarriga@uach.cl

Recibido : 07-11-2018

Aceptado: 25-02-2019 